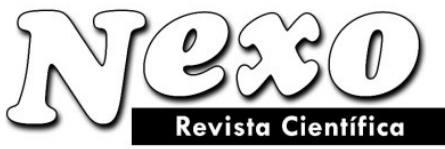

ISSN-E 1995-9516

Universidad Nacional de Ingeniería http://revistas.uni.edu.ni/index.php/Nexo http://dx.doi.org/10.5377/nexo.v30i2.5525

\title{
CARACTERIZACION DE Trichoderma viridae Y T atroviridae AISLADOS DE MONUMENTOS HISTORICOS EN CIUDAD DE MEXICO.
}

\section{CHARACTERIZATION OF Trichoderma viridae AND $T$ atroviridae ISOLATED FROM HISTORICAL MONUMENTS IN MEXICO CITY.}

\author{
L.A. Páramo-Aguilera ${ }^{1, *}$, J.L. Hernández-Mendoza ${ }^{2}$ \\ ${ }^{1}$ Universidad Nacional de Ingeniería. Programa de Investigación Estudios Nacionales y Servicios \\ Ambientales, PIENSA-UNI. Managua, Nicaragua. \\ *1paramo2014@gmail.com \\ ${ }^{2}$ Laboratorio de Biotecnología Experimental. Centro de Biotecnología Genómica. Instituto \\ Politécnico Nacional, Blvd del Maestro s/n Col Narciso Mendoza, Reynosa Tam 88710, México. \\ jhernandezm@ipn.mx
}

(recibido/received: 05-Noviembre-2017; aceptado/accepted: 27-Noviembre-2017)

\section{RESUMEN}

Trichoderma spp. es un hongo polivalente, cuyas propiedades lo postulan como agente de control biológico de fitopatógenos y como estimulador de crecimiento de plantas. Comúnmente aislado de suelos y madera en descomposición, aunque los aislados II2 y II8 ( $T$ viridae y $T$ atroviridae) fueron aislados de monumentos históricos en la Cd de México, DF. Este trabajo presenta la caracterización del antagonismo y promoción de crecimiento vegetal de dichos aislados. Se realizaron pruebas de estos hongos en contra de los hongos fitopatógenos Fusarium oxysporum y Macrophomina phaseolina, aislados de frijol. Los resultados muestran que las especies de Trichoderma en estudio no modifican su velocidad de crecimiento cuando crecen en cultivo dual con los antagonistas. Igual resultado se obtuvo para $F$ oxysporum cuando crece frente a los aislados II8 y II2. M phaseolina incrementa su velocidad de crecimiento de colonia cuando crece en la misma caja de Petri frente a las dos especies de Trichoderma. Se observó antagonismo por competencia por espacio y los mecanismos de antibiosis e hiperparasitismo no fueron detectados en estas pruebas. Al evaluarse el potencial uso agronómico, estimulando el crecimiento en plantas de sorgo a través de la altura y biomasa producida en los diferentes tratamientos se determinó que no tienen la capacidad de estimular el desarrollo de las plantas. Contrariamente, en general se observan efectos inhibitorios del crecimiento y desarrollo de las plantas tratadas. Esto tal vez se deba a que estas cepas se aislaron de monumentos históricos de la zona urbana de la $\mathrm{Cd}$ de México.

Palabras claves: Trichoderma viridae y atroviridae, antagonismo, competencia, estimulación, Sorghum bicolor.

\section{ABSTRACT}

Trichoderma spp. is a polyvalent fungus, whose properties postulate it as a biological control agent of phytopathogens and a plant growth stimulant. Commonly isolated from decaying soils and wood, although isolates II2 and II8 (T viridae and T atroviridae) were isolated from historical monuments in Mexico City, 


\section{L.A. Páramo-Aguilera y J.L. Hernández-Mendoza.}

DF. This work presents the characterization of the antagonism and promotion of plant growth of these isolates. These fungi were tested against phytopathogenic fungi Fusarium oxysporum and Macrophomina phaseolina, isolated from beans. The results show that the Trichoderma species studied do not modify their growth rate when grown in dual culture with the antagonists. The same result was obtained for $F$ oxysporum when it grows against isolates II8 and II2. M phaseolina increases its rate of colony growth when it grows in the same Petri dish compared to the two species of Trichoderma. The antagonism by space competition was observed and mechanisms of antibiosis and hyperparasitism were not detected in these tests. When evaluating the potential agronomic use, stimulating the growth in sorghum

plants through the height and biomass produced in the different treatments was determined that they do not have the capacity to stimulate the development of the plants. In contrast, inhibitory effects of the growth and development are generally observed on the treated plants. This may be due to the fact that these Trichoderma viridae y $T$ atroviridae strains were isolated from historical monuments of the urban area of Mexico City.

Keywords: Trichoderma viridae y atroviridae, antagonism, competence, stimulation, Sorghum bicolor.

\section{INTRODUCCIÓN}

Durante los últimos años, varios investigadores y algunas empresas han mostrado gran interés en estudiar el potencial de Trichoderma como controlador biológico de patógenos del suelo (Tovar, 2008). Los hongos del género Trichoderma spp. son un grupo de microorganismos que habitan naturalmente en suelos agrícolas, con abundante materia orgánica en descomposición y alta densidad de raíces. Trichoderma spp. se encuentra ampliamente distribuido en todo el mundo, además se suelen hallar asociados a la superficie de plantas y cortezas de madera descompuesta, en diferentes zonas de vida y hábitat (Harman et al., 2004). El interés por los hongos de este género, se debe a la capacidad de control ante diversos hongos fitopatógenos y por ende el uso en agricultura. De ellos lo más importante es la competencia por nutrientes o espacio, el mico parasitismo y la antibiosis. La importancia relativa de cada uno de ellos depende de la relación antagonista-patógena y de las condiciones ambientales (HernándezMendoza et al., 2015).

Diversos autores han reportado que Trichoderma induce el crecimiento vegetal al degradar el epispermo de la semilla al producir compuestos que mejoran la germinación y el crecimiento vegetal, ya que acelera el desarrollo de los tejidos meristemáticos primarios, los cuales aumentan el volumen, pelos radiculares, la altura, así como el peso de la planta (Gravel et al, 2007; Shoresh y Harman, 2008a; Shoresh y Harman, 2008b; López-Coria et al., 2016). Este hongo secreta compuestos auxínicos como el ácido Indol Acético, que estimula la germinación, el crecimiento, desarrollo radicular y mejora la asimilación de nutrientes (Hernandez-Mendoza et al., 2012). Todo esto influye en el crecimiento vegetativo de cultivos agrícolas (Harman et al., 2004; Harman, 2006; Gravel et al., 2007; Vinale et al., 2008; Sánchez-Pérez, 2009).

Por otra parte, las enfermedades producidas por hongos fitopatógenos causan pérdidas severas en la agricultura (Rey et al., 2000); estas mermas consisten en la reducción de la calidad y/o la cantidad de la cosecha obtenida (Monte, 2001). La manera tradicional para el control de las enfermedades en cultivos es la aplicación de productos químicos, pero debido a su composición resultan tóxicos e inespecíficos, ya que además de eliminar los organismos fitopatógenos, dañan la flora del suelo (Vinale et al., 2008). Por ello es necesaria la búsqueda de alternativas orientadas al manejo de agentes antagonistas que sean eficientes y compatibles con el ambiente (Hernández-Mendoza et al., 2012). Por otra parte, el hongo Macrophomina phaseolina es capaz de atacar a más de 500 especies de plantas cultivadas, entre ellas sorgo, maíz y fríjol, en las que produce la enfermedad conocida como pudrición carbonosa (Hernández-Mendoza et al., 2015). Además el hongo forma esclerocios como estructuras de resistencia, los que sobreviven en el suelo y son difíciles de destruir (Cardona, 2006). En el caso de Fusarium oxysporum se reporta que ataca a más de 100 especies de plantas gimnospermas y angiospermas (Garofalo and McMillan, 2003).

Nexo Revista Científica / Vol. 30, No. 02, pp. 60-72 / Diciembre 2017 


\section{L.A. Páramo-Aguilera y J.L. Hernández-Mendoza.}

La bioprospección de microorganismos antagonistas como Trichoderma spp. es una de las alternativas actuales para combatir los hongos fitopatógenos. La versatilidad, adaptabilidad y fácil manipulación lo han convertido en uno de los antagonistas más utilizados para el control fitosanitario. El uso agrícola a gran escala de Trichoderma, es una alternativa tecnológica muy eficiente desde el punto de vista productivo y económico para la obtención de biofungicidas de alta calidad, involucra procesos estandarizados con el control de variables y mejorar la cantidad y calidad de las esporas producidas (Hernández-Mendoza et al., 2015).

El objetivo del presente trabajo fue caracterizar agronómicamente a Trichoderma viride cepa II8 y Trichoderma atroviride cepa II2, a través de su antagonismo contra Macrophomina phaseolina y Fusarium oxysporum y analizar su efecto sobre la estimulación de crecimiento vegetal en plántulas de sorgo sembradas en charolas en condición de invernadero.

\section{MATERIALES Y MÉTODOS}

\subsection{Aislamiento de Microorganismos}

Las cepas de Trichoderma (T viridae II8 y $T$ atroviridae II2) son cepas aisladas previamente en monumentos históricos en la ciudad de México, DF e identificadas microbiológica y molecularmente (Páramo-Aguilera et al., 2011; Páramo-Aguilera et al., 2012). Se conservan en el Laboratorio de Biotecnología Industrial del Centro de Biotecnología Genómica del IPN.

La cepa de Macrophomina phaseolina (cepa HMP5) fue aislada de plantas de fríjol (Phaseolus vulgaris) en Cotaxtla, estado de Veracruz, México. La cepa HFO6 de Fusarium oxysporium también se aisló de fríjol en la Isla, estado de Veracruz. Estos materiales fueron proporcionados por el Laboratorio de Biotecnología Vegetal del Centro de Biotecnología Genómica del Instituto Politécnico Nacional.

\subsection{Pruebas de antagonismo}

Los hongos fueron cultivados de manera individual en cajas de Petri empleando medio de cultivo PDA. Se realizaron mediciones del desarrollo de la colonia cada $24 \mathrm{~h}$. Las pruebas de antognismo se realizaron con cultivo dual de Trichoderma spp. contra los fitopatógenos mencionados (Larralde et al., 2008; HernandezMendoza et al., 2012 y 2015) en placas de Petri con PDA. Para ello, se coloca un disco de $7 \mathrm{~mm}$ de diámetro de agar con micelio de siete días de desarrollo de $M$ phaseolina y en el otro extremo, un disco con micelio de $T$ viridae o de $T$ atroviridae de cuatro días de crecimiento. Se realizó el mismo procedimiento para $F$ oxysporium. En estas pruebas se utilizó una cepa de $T$ harzianum como testigo. Cada evaluación fue realizada por triplicado.

\subsection{Estimulación de crecimiento}

La evaluación de la estimulación de crecimiento se realizó usando semillas de sorgo (72 por tratamiento), las cuales fueron inoculadas en una caja de Petri $(55 \mathrm{~mm})$ con $6 \mathrm{~mL}$ de la suspensión de esporas de cada uno de los tratamientos $\left(1 \times 10^{2}\right.$ a $1 \times 10^{6}$ esporas por $\left.\mathrm{mL}^{-1}\right)$. Las semillas se incubaron por $72 \mathrm{~h}$, luego se sembraron en charolas de 72 cavidades (capacidad $120 \mathrm{~mL}$ de sustrato c/u). Para la siembra se utilizó una mezcla de suelo agrícola y perlita en relación $3: 1 \mathrm{v} / \mathrm{v}$. La medición de la estimulación se realizó a los $15 \mathrm{y}$ 30 días después de la siembra en condiciones de invernadero, valorando la biomasa producida (medida como peso seco de raíz, follaje y peso total en gramos) y la altura de la planta (medida como altura de raíz, altura de follaje y altura total en $\mathrm{cm}$ ). El análisis ANOVA se hizo por medio del paquete de procesamiento de datos de EXCEL del programa OFFICE 2013. 


\section{RESULTADOS Y DISCUSIÓN}

\section{1 antagonismo}

Los modos de acción más comúnmente reportadas para Trichoderma spp. Son: micoparasitismo, competencia por nutrientes y espacio; antibiosis, por medio de enzimas o la producción de metabolitos secundarios y micoparasitismo (Harman 2006; Hernandez-Mendoza et al., 2012 y 2015). Una herramienta útil y confiable para conocer el potencial como agente de biocontrol de cepas de Trichoderma son los ensayos in vitro para determinar antagonismo, los cuales se utilizan principalmente como herramienta predictiva para determinar la capacidad de inhibición del crecimiento, antes de efectuar estudios que requieran más tiempo y costo económico (Larralde., et al 2008). Las pruebas de antagonismo que fueron realizadas mediante este trabajo, muestran que las cepas evaluadas tienen una respuesta antagónica de competencia por espacio, ya que el crecimiento de las colonias de fitopatógenos es detenida por el desarrollo de las colonias de Trichoderma spp. Al estudiar la interacción entre $M$ phaseolina y las tres especies de Trichoderma spp, se observa que existe competencia por espacio, el cual es más evidente cuando las colonias tienen 8 días de estar en confrontación (Figura 1).

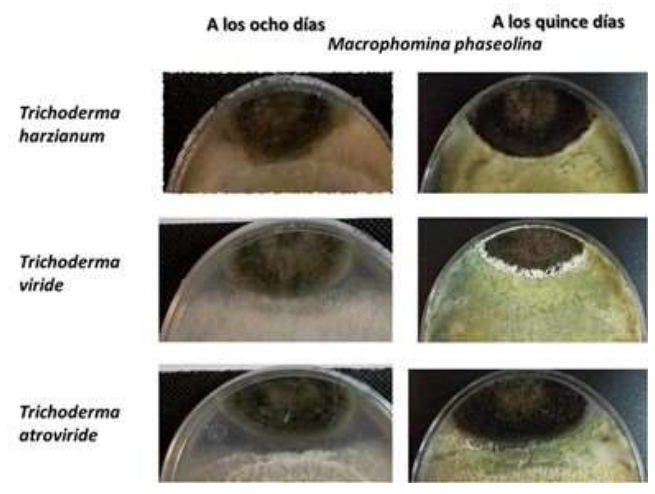

Figura 1. Antagonismo de tipo competencia por espacio de $T$ viridae, $T$ atroviridae y $T$ harzianum contra $M$ phaseolina, ya que al entrar en contacto las colonias de detiene en crecimiento de ambas especies.

En el caso de las evaluaciones hechas en cultivo dual en contra de $F$ oxysporum, se observa que en los primeros 8 días de iniciada la prueba, el antagonismo es por competencia por espacio y cuando las cajas alcanzan los 15 días, $T$ viridae y $T$ atroviridae colonizan y esporulan entre la colonia de $F$ oxysporum (Figura 2). Al realizar observaciones microscópicas no se detectó hiperparasitismo (No se observó que Trichoderma spp parasitara a Fusarium).

A fin de determinar si el crecimiento y desarrollo de los hongos en afectada por el crecimiento en la misma caja de petri, se valoró la cinética de crecimiento de los hongos solos y en confrontación. Solo para el caso de $F$ oxysporum creciendo en antagonismo con $T$ harzianum existe un ligero incremento de la velocidad de desarrollo en las primeras horas con respecto a la condición de crecimiento en individual (Tabla 1, Variante C). A fin de determinar si el crecimiento y desarrollo de los hongos en afectada por el crecimiento en la misma caja de petri, se valoró la cinética de crecimiento de los hongos solos y en confrontación. 


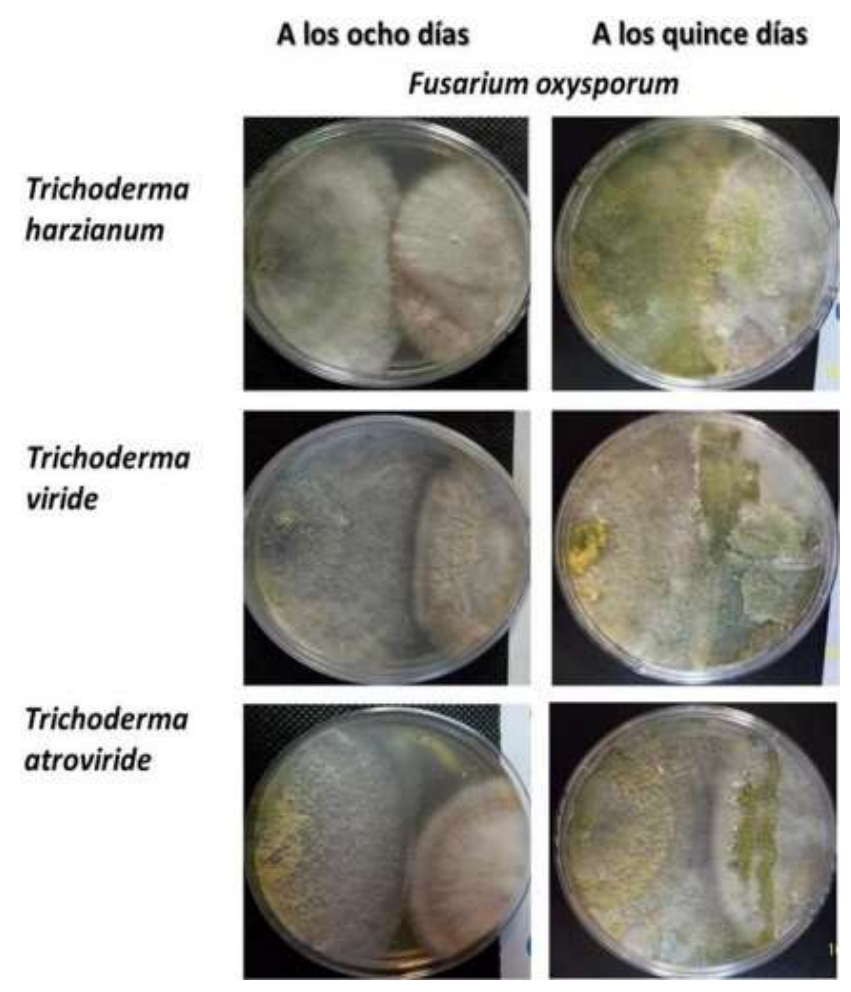

Figura 2. Antagonismo por competencia de $T$ viridae, $T$ atroviridae y $T$ harzianum contra $F$ oxysporum.

Solo para el caso de $F$ oxysporum creciendo en antagonismo con $T$ harzianum existe un ligero incremento de la velocidad de desarrollo en las primeras horas con respecto a la condición de crecimiento en individual (Tabla 1, Variante C). Sin embargo, el análisis ANOVA para los resultados de la confrontación de estos hongos, mostró una $\mathrm{P}=0.2753$, mayor de 0.05 y por ende este ligero incremento de la velocidad de desarrollo de $F$ oxisporum no es estadísticamente significativo en su confrontación con $T$ harzianum. Para el caso de $T$ atroviridae, la cinética de crecimiento es similar a la respuesta obtenida que con $T$ viridae, donde al parecer la presencia de $F$ oxysporum no tiene efecto alguno sobre la velocidad de crecimiento del antagonista. Por su parte, cuando $F$ oxysporum está en confrontación parece en las primeras horas de crecimiento ser estimulado por la presencia de $T$ atroviridae (Tabla 1, Variante B). El análisis ANOVA para la confrontación de $F$ oxysporum contra $T$ viridae produjo una $\mathrm{P}=0.1543$ y contra $T$ atroviridae arroja una $\mathrm{P}=0.2187$, en ambos casos mayor de 0.05 y por ende se comprueban los resultados obtenidos al no haber significancia estadística en cuanto a la cinética de crecimiento en condición de cultivo dual. En general, los resultados de las cinéticas de crecimiento en confrontación de Fusarium contra los diferentes Trichoderma probados en este estudio, no arrojan resultados estadísticamente significativos, lo que se interpreta como que el crecimiento de ambos hongos nos es afectado por la confrontación. 


\section{L.A. Páramo-Aguilera y J.L. Hernández-Mendoza.}

Tabla 1. Cinética de crecimiento de colonias de Trichoderma viridae (Variante A), Trichoderma atroviridae (Variante B) y Trichoderma harzianum (Variante C) creciendo solos y en antagonismo frente a Fusarium oxysporum y Macrophomina phaseolina (Variante A). S: El microorganismo creciendo solo, IF: El microorganismo cuando crece en confrontación con Fusarium, Itv: El microorganismo creciendo en confrontación con Trichoderma viridae, InM: El microorganismo en confrontación con Macrophomina, Ita: El microorganismos en confrontación con Trichoderma atroviridae, Ith: El micoorganismo en confrontación con Trichoderma harzianum.

\begin{tabular}{|c|c|c|c|c|c|c|c|}
\hline $\mathrm{t}(\mathrm{h})$ & $T$ viride $S$ & $\begin{array}{c}\text { viride } \\
I F\end{array}$ & Fusarium $\mathrm{S}$ & $\begin{array}{l}\text { Fusarium } \\
\text { Itv }\end{array}$ & $\begin{array}{c}\mathrm{T} \text { viride } \\
\mathrm{InM}\end{array}$ & $\begin{array}{c}\text { Macrophomina } \\
S \\
\end{array}$ & $\begin{array}{l}\text { Macrophomina } \\
\text { Itv }\end{array}$ \\
\hline 0 & 0 & 0 & 0 & 0 & 0 & 0 & 0 \\
\hline 12 & 0.33 & 0.42 & 0.22 & 0.55 & 0.52 & 0.25 & 0.5 \\
\hline 24 & 0.98 & 1 & 0.43 & 1 & 1.3 & 0.57 & 1 \\
\hline 36 & 2.07 & 2.07 & 0.78 & 1.25 & 2.55 & 0.93 & 2.5 \\
\hline 48 & 3.23 & 2.97 & 1.07 & 1.4 & 3.4 & 1.23 & 3.4 \\
\hline 60 & 4.33 & 3.8 & 1.4 & 1.7 & 4.45 & 1.58 & 3.6 \\
\hline $72 \mathrm{Hr}$ & 5.43 & 4.43 & 1.77 & 2 & 5.1 & 1.8 & 3.7 \\
\hline \multicolumn{8}{|l|}{$\begin{array}{l}\text { Variante } \\
\mathrm{B}\end{array}$} \\
\hline $\mathrm{t}(\mathrm{h})$ & $\begin{array}{c}T \\
\text { atroviride } \\
\mathrm{S}\end{array}$ & $\begin{array}{c}T \\
\text { atroviride } \\
\text { IF }\end{array}$ & Fusarium $\mathrm{S}$ & $\begin{array}{l}\text { Fusarium } \\
\text { Ita }\end{array}$ & $\begin{array}{c}T \\
\text { atroviride } \\
\text { IM }\end{array}$ & $\begin{array}{c}\text { M phaseolina } \\
S \\
\end{array}$ & $\begin{array}{c}\text { M phaseolina } \\
\text { Ita }\end{array}$ \\
\hline 0 & 0.0 & 0 & 0 & 0 & 0 & 0 & 0 \\
\hline 12 & 0.3 & 0.4 & 0.23 & 0.55 & 0.7 & 0.32 & 0.5 \\
\hline 24 & 0.8 & 1.1 & 0.48 & 1 & 1.55 & 0.67 & 1 \\
\hline 36 & 1.7 & 1.97 & 0.75 & 1.25 & 2.7 & 1.03 & 2.5 \\
\hline 48 & 2.8 & 2.82 & 1.02 & 1.44 & 3.42 & 1.53 & 3.4 \\
\hline 60 & 3.6 & 3.63 & 1.42 & 1.7 & 4.42 & 1.75 & 3.6 \\
\hline $72 \mathrm{Hr}$ & 4.7 & 4.1 & 1.73 & 2 & 4.9 & 1.97 & 3.7 \\
\hline \multicolumn{8}{|l|}{$\begin{array}{l}\text { Variante } \\
\mathrm{C}\end{array}$} \\
\hline $\mathrm{t}(\mathrm{h})$ & $\begin{array}{c}T \\
\text { harzianum } \\
S \\
\end{array}$ & $\begin{array}{c}T \\
\text { harzianum } \\
\text { IF } \\
\end{array}$ & Fusarium $S$ & $\begin{array}{c}\text { Fusarium } \\
\text { Ith }\end{array}$ & $\begin{array}{c}T \\
\text { harzianum } \\
\text { IM } \\
\end{array}$ & $\begin{array}{c}\text { Macrophomina } \\
S \\
\end{array}$ & $\begin{array}{c}\text { Macrophomina } \\
\text { Ith }\end{array}$ \\
\hline 0 & 0 & 0 & 0 & 0 & - & - & 0 \\
\hline 12 & 0.37 & 0.32 & 0.23 & 0.55 & 0.30 & 0.20 & 0.5 \\
\hline 24 & 0.83 & 0.9 & 0.5 & 1 & 1.00 & 0.53 & 1 \\
\hline 36 & 1.42 & 2.02 & 0.77 & 1.25 & 2.10 & 1.07 & 2.5 \\
\hline 48 & 2.37 & 3.02 & 1.13 & 1.44 & 3.03 & 1.33 & 3.4 \\
\hline 60 & 3.2 & 3.57 & 1.48 & 1.7 & 4.27 & 1.67 & 3.6 \\
\hline $72 \mathrm{Hr}$ & 4.25 & 4.2 & 1.8 & 2 & 5.23 & 1.88 & 3.7 \\
\hline
\end{tabular}

Nexo Revista Científica / Vol. 30, No. 02, pp. 60-72 / Diciembre 2017 


\section{L.A. Páramo-Aguilera y J.L. Hernández-Mendoza.}

En las pruebas de antagonismo entre $T$ viridae y $M$ phaseolina, la colonia de $T$ viridae se desarrolla a la misma velocidad sola o en antagonismo. Al contrario, cuando $M$ phaseolina crece en confrontación, la velocidad de crecimiento se incrementa apreciablemente, igual que en el caso anterior (Tabla 1, Variante A). Por su parte, en la confrontación de T harzianum contra $M$ phaseolina, se observó que el primero se desarrolla de forma similar en presencia o en ausencia del fitopatógeno. Por el contrario, $T$ harzianum al parecer si tiene un efecto sobre la estimulación del crecimiento de $M$ phaseolina, pues hay un incremento en la velocidad de crecimiento de la colonia cuando $T$ harzianum está presente. Probablemente el antagonista produzca volátiles que causen la aceleración del desarrollo en el fitopatógeno (Tabla 1, Variante C). A partir de los datos mostrados (Tabla 1), las cepas probadas de $T$ viridae (Variante A) y $T$ atroviridae (Variante B), al igual que el testigo T harzianum (Variante $\mathrm{C}$ ), estimulan el crecimiento de $M$ phaseolina, tal por la emisión de compuestos volátiles que liberen las colonias durante su desarrollo (Lee et al., 2016; Tabarestani et al., 2016). Sin embargo el resultado de los análisis ANOVA para cada uno de los procesos de confrontación anteriores muestran que no son significativos ya que para Macrophomina versus $T$ viridae se obtiene una $\mathrm{P}=0.2989>0.05$, contra $T$ atroviridae una $\mathrm{P}=0.3505$ y contra $T$ harzianum se obtuvo una $\mathrm{P}=0.4023$, en todos los casos mayor de 0.05 . Por tanto los incrementos en la acción sobre la cinética del patógeno por parte Trichoderma resultan ser no significativos y por lo tanto, en este caso, los aislados de Trichoderma spp no estimulan el desarrollo y crecimiento de las plantas tratadas.

Ladino-Rey et al., 2017, mostraron la acción inhibidora de cepas de Trichoderma viridae frente el cepas de Fusarium spp, aunque estos autores no lograron determinar la especie de Fusarium con la que trabajaron. Gajera et al., 2013, demostraron la efectividad de las especies del genero Trichoderma spp debido a sus diferentes mecanismos de acción, tales como micoparasitismo, competencia por espacio, antibiosis e inducción a resistencia sistémica. Los resultados de este trabajo permiten comprobar que Trichoderma viridae y atroviridae ejercen una acción de inhibición del crecimiento sobre los fitopatógenos Fusarium oxysporun y Macrophomina phaseolina, que se describe como una competencia por espacio y no se puede evidenciar el micoparasitismo. Por otra parte, el incremento en la cinética del crecimiento que se observa en el caso de los fitopatógenos en su confrontación con Trichoderma, resulta ser no significativa y estadísticamente comprobada por medio del análisis ANOVA, lo cual se puede interpretar como la ausencia de volátiles que estimulen el desarrollo de los fitopatógenos evaluados.

\subsection{Estimulación de crecimiento en sorgo}

Trichoderma posee la capacidad de estimular el crecimiento de la planta y producir auxinas que están relacionadas al desarrollo del sistema radical que aumenta la tolerancia de la planta al estrés (HernandezMendoza et al., 2012; Mukherjee et al., 2013). Dichos efectos se han observado en la colonización de $T$. virens en la rizósfera del maíz, el cual induce a un aumento en la tasa de la fotosíntesis, así como en los niveles de captación de $\mathrm{CO}_{2}$ en las hojas (Vargas et al., 2009). Estudios de Mastouri et al. (2010) reportaron aumento en la germinación de semillas de tomate al ser estas tratadas con T. harzianum, este mismo efecto es observado en otras plantas debido a la simbiosis que establecen con especies de Trichoderma (Vadassery et al., 2009).

Las evaluaciones realizadas mediante este trabajo muestran que las dos cepas probadas, correspondientes a $T$ viridae y $T$ atroviridae, no inhiben la germinación ni el desarrollo de las semillas tratadas. Las semillas germinan en la caja Petri aunque el micelio del hongo haya cubierto la totalidad de la superficie disponible. En plantas germinadas, las cepas de Trichoderma pueden esporular en la testa de la semilla sin que el crecimiento y desarrollo de la planta sea afectada de manera negativa (Figura 3). Se pudo observar que a los tres días de la inoculación, las placas inoculadas con Trichoderma viridae (cepa II2) presentan un porcentaje de germinación entre el 60 y el $70 \%$, coincidiendo con el testigo negativo. En cambio Trichoderma atroviridae (cepa II8) presenta de 80-90 \% de germinación. Lo anterior conduce a una 


\section{L.A. Páramo-Aguilera y J.L. Hernández-Mendoza.}

estimulación de la germinación por parte de T atroviridae en condiciones de placa Petri. El análisis de los resultados de la germinación a los seis días, muestran que T.viridae estabiliza su porcentaje de germinación en un $70 \%$, lo cual está por debajo del testigo negativo que alcanzo hasta un $80 \%$. Por otra parte $T$ atroviridae, alcanzó hasta un $90 \%$ de germinación, confirmando la estimulación por parte de este último al proceso de germinación de la semilla de sorgo.

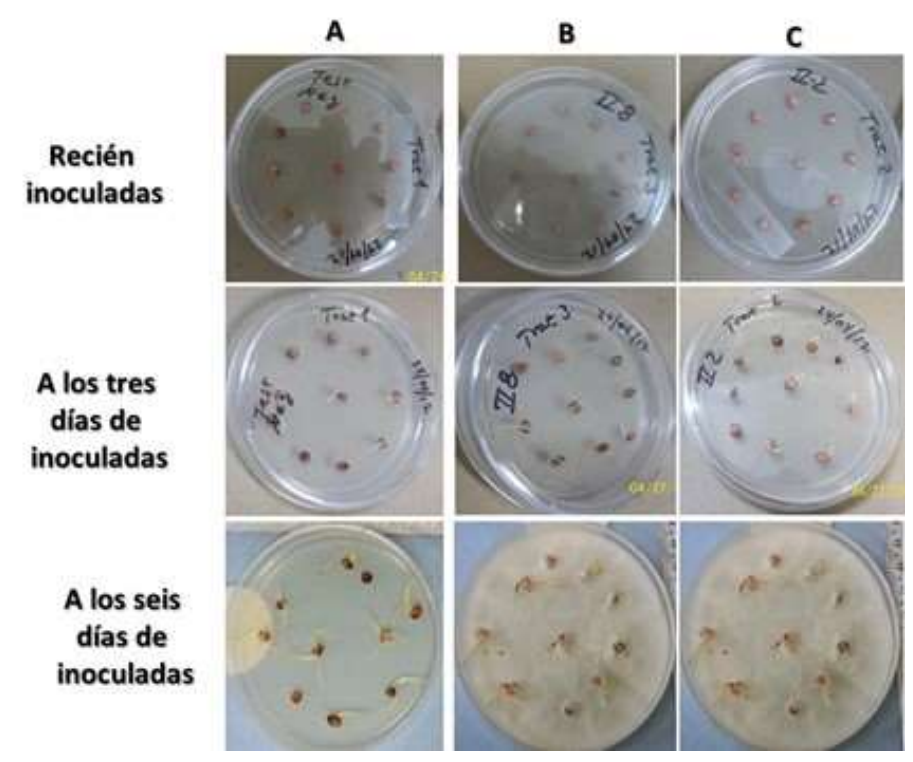

Figura 3: Seguimiento a la inoculación de semillas de sorgo, mostrando la ausencia de fitopatogenicidad de las diferentes especies de Trichoderma spp. A. Testigo negativo. B. Cepa II.8 correspondiente a Trichoderma atroviridae y C. Cepa II.2 correspondiente a Trichoderma viridae.

Si se considera que ambas cepas utilizadas en este estudio fueron aisladas de monumentos históricos de la ciudad de México, las diferencias en su capacidad de estimular el proceso de germinación para la semilla de sorgo bien pueden explicarse por las diferencias en cuanto al hábitat de las especies utilizadas y su aplicación a nivel de invernadero, esto ha sido muy bien demostrado por diversos autores (Mastouri et al., 2010).

El seguimiento al proceso a nivel de invernadero permitió analizar el efecto de ambos aislados en estudio y medirlo por medio del desarrollo de la biomasa como se muestra en las Tablas 2 y 3, así como en las Figuras 4 y 5 . El análisis de la tabla 2, nos muestra que a los 15 días de la siembra a nivel de charola se produce una ligera estimulación en la longitud de raíz (Tabla 3 y figura 5), pero este efecto es inverso cuando se analiza la altura de planta y se obtienen mayores valores para el testigo negativo que los obtenidos con Trichoderma. El análisis ANOVA para los resultados de altura de raíz a los 15 días, indica que no hay diferencias significativas para los valores obtenidos con los diferentes tratamientos ya que $\mathrm{P}=$ $0.4043>0.05$. El mismo parámetro medido a los 30 días muestra que $\mathrm{P}=0.3185>0.05$, manteniendo el resultado la misma condición de no significativos a los 30 días. Por tanto con respecto a la longitud de raíz, ninguno de los aislados probados ( $T$ viridae y $T$ atroviridae) ejercen ninguna influencia en comparación a los resultados cuando no se aplica ningún tipo de hongo.

La misma Tabla 2, nos muestra los resultados de la altura de planta (follaje) a los 15 y 30 días respectivamente, a simple vista se obtiene que el testigo negativo aporta plantas con mayor altura para ambos momentos analizados. Los resultados del ANOVA para esta variable a los 15 días aportan una $\mathrm{P}=$ $8.55107 \mathrm{E}-04<0.05$ y a los 30 días una $\mathrm{P}<7.42756 \mathrm{E}-05$, lo cual indica que en ambos momentos de análisis se obtienen diferencias significativas. Lo anterior nos muestra un efecto inhibitorio en el 


\section{L.A. Páramo-Aguilera y J.L. Hernández-Mendoza.}

crecimiento de la planta provocado por ambos aislados en estudio y confirma lo que antes se dijo de la simple observación de los datos.

Tabla 2. Evaluación de la longitud de raíz y altura de planta de sorgo tratadas con $T$ viridae cepa II2 y $T$ atroviridae cepa II8 en sorgo a los 15 y 30 días de iniciado el experimento a nivel de invernadero.

$\begin{array}{llcc}\text { Variantes } & \text { Long de Raíz }(\mathrm{cm}) & \text { Alt planta }(\mathrm{cm}) & \text { Altura total }(\mathrm{cm}) \\ \text { Test. Neg. 15d } & 27.75 & 35.31 & 63.06 \\ \text { Cepa II8 15 d } & 29.18 & 32.81 & 62 \\ \text { Cepa II2 15d } & 29.69 & 31.76 & 61.46 \\ \text { Test. Neg. 30d } & 29.17 & 39.7 & 68.88 \\ \text { Cepa II8 30 d } & 30.62 & 34.68 & 65.31 \\ \text { Cepa II2 30d } & 30.86 & 36.2 & 67.06\end{array}$

La figura 4, muestra el comportamiento del peso seco de follaje y de raíz a los 15 días de tratadas con ambas cepas de Trichoderma spp en estudio. Aparentemente a los 15 días la cepa II8 ( $T$ atroviridae) presenta mejores resultados ante sorgo que II2 ( $T$ viridae), pero ambos arrojan resultados por debajo de los obtenidos con el testigo negativo en lo referido a peso de follaje, o sea cuando no se adiciona ningún Trichoderma. Solamente se observó un ligero incremento del peso seco de raíz cuando se usa la cepa II8 en relación a cuando no se usó nada (testigo negativo, Tabla 3), lo cual está en aparente relación con lo antes discutido y con lo observado por medio de la figura 5 para la misma cepa.

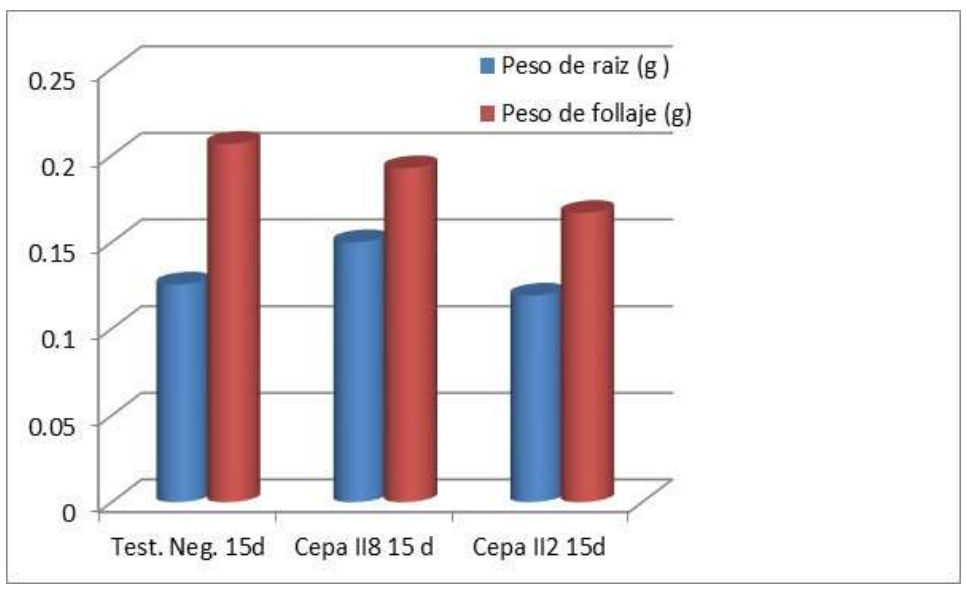

Figura 4. Peso seco de follaje y de raíz de plantas de sorgo a 15 días de tratadas con $T$ viridae cepa II2, T atroviridae cepa II8 y testigo sin inocular. Similar comportamiento se observó con la medición de estas variables a los 30 días.

El análisis ANOVA para el peso de raíz a los 15 días $(\mathrm{P}=0.1907>0.05)$ y a los 30 días $(\mathrm{P}=4.65744 \mathrm{E}-$ $04<0.05)$ nos muestran que a los 15 días no hay diferencia significativa en cuanto al peso de raíz para ninguno de los tratamientos, sin embargo a los 30 días se observa que las diferencias en los resultados hacen que sean significativos, mostrando que el efecto inhibitorio en el crecimiento de la planta que está reflejado en el peso de la raíz después de los 15 días y se ve claramente a los 30 días, razón por la cual el testigo negativo aporta mejores resultados (Tabla 3). Para la variable peso de follaje a los 15 días $(\mathrm{P}=$ $0.1716>0,05)$ y a los 30 días $(\mathrm{P}=1.1683 \mathrm{E}-03<0.05)$. Estos resultados concuerdan perfectamente con lo antes discutido y reafirman el efecto inhibitorio que ejercen tanto $T$ atroviridae como $T$ viridae en el crecimiento y desarrollo de la planta, afectando no solo el desarrollo del sistema radicular, sino también su peso seco a nivel de raíz y follaje. 


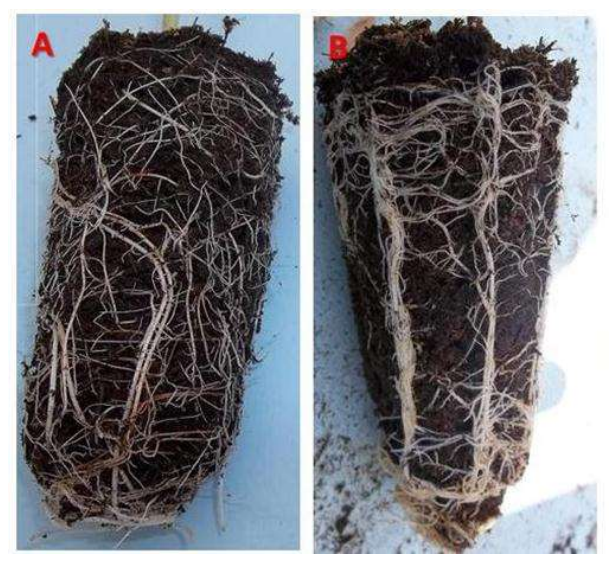

Figura 5. Desarrollo del sistema radicular y plántulas de sorgo a nivel de invernadero con cepa II8. A. Desarrollo a los 15 días de inoculación. B. Desarrollo a los 30 días de inoculación.

Se ha reportado que Trichoderma induce el crecimiento vegetal al degradar el epispermo de la semilla e intervenir en los procesos respiratorios durante la germinación. Además, acelera el desarrollo de los tejidos meristemáticos primarios, los cuales aumentan el volumen, la altura, así como el peso de la planta (Gravel et al, 2007; Shoresh y Harman, 2008a; Shoresh y Harman, 2008b; López-Coria et al., 2016). La tabla 3 permite un análisis completo de los resultados obtenidos a los treinta días en invernadero, y se puede observar que para este tiempo ya ninguna de las cepas en estudio supera al testigo negativo en lo que se refiere a peso de raíz y peso de follaje, por tanto es posible suponer que tanto $T$ atroviridae, como $T$ viridae inhiben en el tiempo el crecimiento y desarrollo en general de la planta de sorgo a nivel de invernadero. Este resultado, contrario a lo antes reportado, abre una nueva interrogante en cuanto a la especificidad de las cepas de Trichoderma con respecto al cultivo con el que se asocien, su región de procedencia y las condiciones ambientales.

Algunos autores como Harman et al., 2004, han sugerido la posibilidad de que sean algunas cepas seleccionadas de Trichoderma quienes promuevan el crecimiento y desarrollo de las plantas y por tanto el rendimiento de las cosechas. Por otra parte, Arispe (2014), menciona la incidencia del tipo de Trichoderma sobre el rendimiento en cultivos de maíz y lo mismo fue corroborado por medio de su trabajo de investigación.

Tabla 3. Evaluación de peso seco de raíz y de follaje de la planta de sorgo tratada con $T$ viridae cepa II2 y $T$ atroviridae cepa II8 a los 15 y 30 días de iniciada la evaluación.

\begin{tabular}{lccc}
\hline Variantes & Peso de raíz $(\mathrm{g})$ & Peso de follaje $(\mathrm{g})$ & Peso total $(\mathrm{g})$ \\
\hline Test. Neg. 15d & 0.1256 & 0.2068 & 0.3325 \\
Cepa II8 15 d & 0.15 & 0.1927 & 0.3427 \\
Cepa II2 15d & 0.1192 & 0.1669 & 0.2861 \\
& & & \\
Test. Neg. 30d & 0.4864 & 0.7052 & 1.1917 \\
Cepa II8 30 d & 0.3618 & 0.4975 & 0.8593 \\
Cepa II2 30d & 0.3726 & 0.4553 & 0.858 \\
\hline
\end{tabular}

\section{CONCLUSIONES}

Los resultados obtenidos por medio de este trabajo permitieron demostrar que Trichoderma viridae y atroviridae ejercen una acción de inhibición del crecimiento sobre los fitopatógenos Fusarium oxysporun y Macrophomina phaseolina que se describe como una competencia por espacio sin que se puedan 
evidenciar rasgos de micoparasitismo. Por otra parte, el incremento en la cinética del crecimiento que se observa en el caso de los fitopatógenos en su confrontación con Trichoderma, resulta ser no significativa y estadísticamente comprobada por medio del análisis ANOVA. $T$ atroviridae resultó estimular el proceso de germinación de semillas de sorgo (a nivel de placa Petri ) previamente impregnadas con esporas del mismo al alcanzar un $10 \%$ más de germinación por encima del testigo negativo y $T$ viridae arrojó resultados inferiores al testigo negativo, por lo que pudiera estar inhibiéndose la germinación del sorgo por parte de $T$ viridae. Los análisis estadísticos por medio del ANOVA permitieron comprobar el efecto inhibitorio en el desarrollo de las plántulas de sorgo para todo el tiempo de análisis y al mismo tiempo muestran que ninguno de los aislados de Trichoderma en evaluación ejerce influencia significativa en el desarrollo del sistema radicular. Quedó comprobado que tanto se inhibe el sistema radicular como el follaje, afectando de esta forma el peso seco tanto de raíz como de follaje. Estas diferencias con respecto a lo reportado hasta la fecha, bien pudieran estar relacionadas con el hábitat de los aislados y su procedencia a partir de monumentos históricos.

\section{REFERENCIAS}

Arispe, V.J.A. (2014). Biocontrol de la pudrición de mazorca causada por Fusarium sp. En cuatro genotipos de maíz con Trichoderma spp bajo condiciones de campo en la región de Tepalcingo. Morelos. Universidad Autónoma Agraria Antonio Narro. Presentada como requisito parcial para obtener el título de Ingeniero Agrónomo Parasitólogo. Saltillo, Coahuila, México. 2014.

Cardona, R. (2006). Distribución vertical de esclerocios de Macrophomina phaseolina en un suelo infestado naturalmente en el estado Portuguesa. Rev. Fac. Agron. (LUZ) 23: 284-29151.

Gajera H., Rinkal D., Patel S., Kapopara M., Golakiya B. (2013). Molecular mechanism of Trichoderma as bio-control agents against phytopathogen system-a review. Current Research in Microbiology and Biotechnology 1:133-142.

Garofalo, J. y McMillan, R. T. Jr. (2003). Marchitez por Fusarium en palmas del género Phoenix del sur de Florida. Hoja informativa No. 96, Miami-Dade County/University of Florida Cooperative Extension Service. Traducido al español por Delgado R. y Balerdi C. en 11/2003.

Gravel, V., Antoun, H. and Tweddell, R. J. (2007). Growth stimulation and fruit yield improvement of greenhouse tomato plants by inoculation with Pseudomonas putida or Trichoderma atroviride: Possible role of indole acetic acid (IAA). Soil Biol. Biochemist. 39: 1968-1977.

Harman, G.E., C.R. Howell, A. Viterbo, I. Chet, and M. Lorito. (2004). Trichoderma species opportunistic, avirulent plant symbionts. Nature Reviews Microbiology 2:43-56.

Harman, G. E. (2006). Overview of mechanisms and uses of Trichoderma spp. Phytopathology 96: 190194.

Hernández-Mendoza J. L., Quiroz-Velázquez J. D., Díaz-Franco A., García-Olivares J. G., BustamanteDávila A. J. and Gill-Langarica H. R. (2012). Detection of metabolites in Flor de Mayo common beans (Phaseolus vulgaris L.) and their response to inoculation with Trichoderma harzianum. African Journal of Biotechnology. Vol. 11(55): 11767-11771.

Hernandez-Mendoza, JL, María Isabel Sánchez Pérez, Juan Manuel González Prieto, Jesús DiCarlo Quiroz Velásquez, Jesús Gerardo García Olivares, Homar Rene Gill Langarica. (2015). Antibiosis of Trichoderma spp strains native to northeastern Mexico against the pathogenic fungus Macrophomina phaseolina. Brazilian Journal of Microbiology 46, 4, 1093-1101.

Nexo Revista Científica / Vol. 30, No. 02, pp. 60-72 / Diciembre 2017 
Ladino-Rey, O.E., Chavez-Betancourt, G.A., Rubio-Gómez, J.D y Chacin-Zambrano, Ch.A. (2017). Evaluación de Trichoderma sobre hongos contaminantes de semillas de Palma hibrida interespecífica (Elaeis oleifera $x$ Elaeis guineensis). Respuestas. 22(1), pp: 96-102, https://doi.org/10.22463/0122820X.859.

Larralde, C.C., Santiago, M.R., Sifuentes, R.A., Rodríguez, L.I., Rodríguez, P.M and Narváez, Z. J. (2008). Biocontrol potential and polyphasic characterization of novel native Trichoderma strain against Macrophomina phaseolina isolates from sorghum and common bean. Applied Microbiology and Biotechnology 80: 167-177.

Lee, S, Melanie Yap, Gregory Behringer, Richard Hung and Joan W. Bennett. (2016). Volatile organic compounds emitted by Trichoderma species mediate plant growth. Fungal Biol Biotechnol (2016) 3:

Mastouri, F., Bjorkman, T., Harman, G.E. (2010). Seed treatments with Trichoderma harzianum alleviate biotic, abiotic and physiological stresses in germinating seeds and seedlings. Phytopathology 100:12131221.

Monte, E. (2001). Understanding Trichoderma: Between biotechnology and microbial ecology. Int. Microbiol 4: 1-4.

Mukherjee, P.K., Horwitz, B.A., Herrera-Estrella, A., Schmoll, M., Kenerley, C.M. (2013). Trichoderma Research in the Genome Era. Annu. Rev. Phytopathol. 51:105-29.

Páramo-Aguilera, L.A., Narváez, Z.J y De la Cruz, E. (2011). Aislamiento e identificación de microorganismos en biopelículas provenientes del Castillo de Chapultepec, Ciudad de México. Revista cientifica NEXO, Vol.24, $\mathrm{N}^{0} .02$, pp.83-91.

Páramo-Aguilera, L.A., Ortega-Morales, B.O and Narváez-Zapata, J.A. (2012). Culturable fungi associated with urban Stone surfaces in Mexico City. Electronic Journal Biotechnology. Vol.15. N ${ }^{0} .4$. DOI: 10.2225/vol15-issue4-fulltext-6.

Rey, M., Delgado, J. J., Rincón, A. M., Limón, M. C. y Benítez, T. (2000). Mejora de cepas de Trichoderma para su empleo como biofungicidas. Rev. Iberoam Micol 17: S31-S36.

Sánchez-Pérez, M. I. (2009). Aislamiento y caracterización molecular y agronómica de Trichoderma spp. Nativos del norte de Tamaulipas. Tesis de Maestría en Ciencias en Biotecnología Genómica. Centro de Biotecnología Genómica. Instituto Politécnico Nacional, 187 p.

Shoresh, M. y Harman, G. E. (2008a). The molecular basis of shoot responses of maize seedlings to Trichoderma harzianum T22 inoculation of the root: A proteomic approach. Plant Physiology. 147: 21472163.

Shoresh, M. y Harman, G. E. (2008b). The relationship between increased grow and resistance induced in plants by root colonizing microbes. Plant Signaling \& Behavoir. 3: 737-739.

Tabarestani, M Shahiri, K. Rahnama, M. Jahanshahi, S. Nasrollahnejad, M. H. Fatemi. (2015). Identification of volatile organic compounds of Trichoderma spp. using static headspace gas chromatography-mass spectrometry. Mycologia Iranica. 3(1): $47-55$.

Tovar, C.J.C. (2008). Evaluación de la capacidad antagonista "In vivo" de aislamientos de Trichoderma sp frente al hongo fitopatógeno Rhizoctonia solani. Pontificia Universidad Javeriana de Colombia. Trabajo de grado para obtener el título de Microbiólogo agrícola y veterinario. 
Vadassery, J., Tripathi, S., Prasad, R., Varma, A., Oelmuller, R. (2009). Monodehydroascorbate reductase 2 and dehydroascorbate reductase 5 are crucial for a mutualistic interaction between Piriformospora indica and Arabidopsis. J. Plant Physiol. 166: 1263-1274.

Vargas W.A., Mandawe J.C., Kenerley C.M. (2009). Plant-derived sucrose is a key element in the symbiotic association between Trichoderma virens and maize plants. Plant Physiology. 151:792-808.

Vinale, F., Sivasithamparamb, K., Ghisalbertic, M. L., Marra, R., Woo, S. L., Lorito, M. (2008). Trichoderma-plant-pathogen interactions. Soil Biology \& Biochemistry 40: 1-10.

\section{SEMBLANZA DE LOS AUTORES}

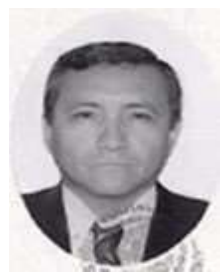

Leandro Alberto Páramo Aguilera, Graduado como Ingeniero Químico con maestría en Ingeniería Química y énfasis en procesos biotecnológicos, en el Instituto Superior Politécnico "José Antonio Echeverría”, ISPJAE, de la Ciudad de la Habana, Cuba, en el año 1990. En 1997 se gradúa como Master en Microbiología y énfasis en bacteriología en la Universidad de Costa Rica, UCR. En junio del 2012, se gradúa como Doctor en Ciencias en el área de Biotecnología en el Centro de Biotecnología Genómica (CBG) del Instituto Politécnico Nacional (IPN) de México. Amplia experiencia en el desarrollo de procesos biotecnológicos (biofertilizantes, bebidas alcohólicas, fermentados lácteos, bioprospección, compostaje, etc).

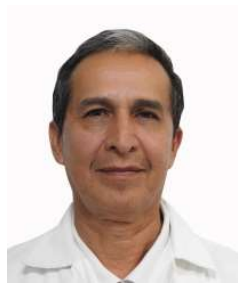

José Luis Hernández Mendoza: Obtuvo el grado de Biólogo en la Universidad Autónoma de Nuevo León, México. El Doctorado en la Universidad de Ciencias y Técnicas de Languedoc, Francia. Actualmente es profesor titular C y responsable del Lab de Biotecnología Experimental en el Centro de Biotecnología Genómica del Instituto Politécnico Nacional; miembro del Sistema Nacional de Investigadores nivel 1. Ha desarrollado estancias de investigación en el Instituto Pasteur de Paris y el Instituto Nacional de Investigaciones Agrícolas, ambos en Francia. También en el Citrus Center de la Universidad de Texas A\&M unidad Kingsville, Estados Unidos. Trabaja en investigación sobre uso y manejo de inoculantes en agricultura, donde ha hecho desarrollos tecnológicos en biofertilizantes que se usan actualmente en el norte de México. 